\title{
Zero-temperature hysteresis in a random-field Ising model on a Bethe lattice: Approach to mean-field behavior with increasing coordination number $z$
}

\author{
Xavier Illa, ${ }^{1, *}$ Prabodh Shukla, ${ }^{1,2}$ and Eduard Vives ${ }^{1}$ \\ ${ }^{1}$ Departament d'Estructura i Constituents de la Matèria, Universitat de Barcelona Diagonal 647, Facultat de Física, \\ 08028 Barcelona, Catalonia, Spain \\ ${ }^{2}$ Physics Department, North Eastern Hill University, Shillong-793022, India
}

(Received 16 November 2005; published 24 March 2006)

\begin{abstract}
We consider the analytic solution of the zero temperature hysteresis in the random field Ising model on a Bethe lattice of coordination number $z$, and study how it approaches the mean-field solution in the limit $z$ $\rightarrow \infty$. Analytical results concerning the energy of the system along the hysteresis loop and first-order reversal curves (FORC diagrams) are also presented.
\end{abstract}

DOI: 10.1103/PhysRevB.73.092414

PACS number(s): 75.60.Ej, 75.10.Nr, 05.50.+q, 75.40.Mg

The random field Ising model with zero temperature $(T$ =0) metastable dynamics is a prototype lattice model for understanding the dynamics of disorder driven first-order phase transitions and hysteresis in several complex systems. ${ }^{1}$ The model was introduced more than 10 years ago and studied analytically in the mean-field limit and numerically on finite dimensional lattices (three-dimensional (3D), four dimensional (4D), etc.). It predicts the existence of a critical point in the systems's response to a slowly and smoothly varying applied field. Theoretical interest in the model increased after many of its properties were obtained analytically on Bethe lattices. ${ }^{2}$ It was found that the critical point exists only on lattices of coordination number $z \geqslant 4$. In recent years the analysis on Bethe lattices has been extended to obtain additional results like the trajectories of the first-order reversal curves in the field versus magnetization $(\mathrm{H}-\mathrm{m})$ diagram, ${ }^{3}$ the behavior of the different energy terms in the Hamiltonian $^{4}$ and the demagnetized states. ${ }^{5}$ In this Brief Report we present developments along three lines: (i) Relationship between the mean-field solution of the model and its solution on Bethe lattices of large coordination numbers, (ii) a different argument for the computation of the energy terms that allows us to rewrite the existing results for a $z=4$ lattice in a more compact and transparent form valid for an arbitrary value of $z$, and (iii) the computation of the FORC-diagrams that allow a compact description of the properties of the firstorder reversal curves (FORC).

The RFIM model in the mean-field limit of infinetely weak but infinitely long range pair interactions is described by the Hamiltonian,

$$
\mathcal{H}^{M F}=-\frac{J}{N-1} \sum_{i, j} S_{i} S_{j}-\sigma \sum_{i} h_{i} S_{i}-H \sum_{i} S_{i}
$$

Here $J$ is a ferromagnetic exchange interaction of order unity, $\left\{S_{i}= \pm 1 ; i=1,2, \ldots N\right\}$ denote Ising spins, $\left\{h_{i}\right\}$ are independent, identically distributed, on-site quenched random fields, and $H$ is an externally applied uniform field. The quenched fields are drawn from a Gaussian distribution $\phi\left(h_{i}\right)$ of variance unity and mean value zero. The sums over $i$ run over all sites of the system, and the sum over $j$ runs over the entire $N-1$ spins of the system that interact with the spin $S_{i}$.
The factor $N-1$ dividing $J$ ensures that the energy of the system is an extensive quantity.

The model on a Bethe lattice (deep interior of an infinite Cayley tree of coordination number $z$ ) is based on short range interactions. It is characterized by the Hamiltonian,

$$
\mathcal{H}^{B}=-\frac{J}{z} \sum_{i, j} S_{i} S_{j}-\sigma \sum_{i} h_{i} S_{i}-H \sum_{i} S_{i}
$$

The difference between the two look alike Hamiltonians lies in the sum over $j$. In Eq. (2), the sum over $j$ runs over $z$ nearest neighbors of each site $i \quad(z=2 d$, for a $d$-dimensional cubic lattice). The factor $z$ dividing $J$ in Eq. (2) ensures that the energy remains extensive in the limit $z \rightarrow N$. Eqs. (1) and (2) appear to have an identical form if $z=N-1$, but the topology is different in the two cases. In mean-field theory every spin interacts with every other spin but this is not the case on a Bethe lattice.

In both cases (MF and $\mathrm{B}$ ) the local force on a site can be writen in terms of a generic local magnetization $m_{i}$

$$
F_{i}=J m_{i}+\sigma h_{i}+H
$$

where in the MF case $m_{i}^{M F}=m^{M F}=\sum_{j=1}^{N} S_{j} / N$ (except for a negligible small correction in the thermodynamic limit) and for the B case $m_{i}^{B}=\sum_{j=1}^{z} S_{j} / z$. To fully specify the metastable $T=0$ dynamics one must fix the initial state (for instance, $S_{i}=-1$ at $\left.H=-\infty\right)$ and adiabatically sweep the field $H$, relaxing the spins according to the rule $S_{i}=\operatorname{sign}\left(F_{i}\right)$. In the MF description, since $m_{i}$ is independent of $i$, the evolution of the magnetization $m^{M F}(H)$ is trivially determined by the two coupled equations,

$$
\begin{gathered}
m^{M F}=2 p^{M F}-1 \\
p^{M F}=\int_{-J m^{M F}-H / \sigma}^{\infty} \phi\left(h_{i}\right) d h_{i}
\end{gathered}
$$

where the first equation relates the magnetization with the probability $p^{M F}$ of finding a spin up and the second one expresses that probability as an integral of the distribution of random fields. The above equations [Eqs. (4) and (5)] admit one fixed point solution for all fields if $\sigma>\sigma_{c}=\sqrt{\frac{2}{\pi}} J$ and 
three fixed point solutions (in a certain field range) below $\sigma_{c}$. Two of the solutions are stable and one is unstable. The two stable solutions are obtained by numerical iteration starting from the initial state $m=-1$ and $m=1$, respectively, and correspond to the two halves of the hysteresis loop in increasing and decreasing applied field. Note that there is no hysteresis if $\sigma>\sigma_{c}$. The critical point $\left(H_{c}=0, \sigma_{c}\right)$ corresponds to a nonequilibrium critical point of the system. ${ }^{1}$ The Bethe lattice allows short range fluctuations in the environment of each site. Therefore, $m_{i}$ is not homogeneous and can take $z+1$ different values $(-1,(-z+2) / z, \ldots,(z-2) / z, 1)$. Let us introduce a variable $k=0, \ldots z$ indexing the environments so that $m_{i}=(-z+2 k) / z$. To find the behavior of the magnetization $m^{B}$ (for instance, along the $H$-upwards branch of the hysteresis loop) one should treat separately each of these environments. The coupled equations become slightly more involved: ${ }^{2}$

$$
\begin{gathered}
m^{B}=2 p^{B}-1 \\
p^{B}=\sum_{k=0}^{z}\left(\begin{array}{l}
z \\
k
\end{array}\right)\left[P^{*}\right]^{k}\left[1-P^{*}\right]^{z-k} P\left(S_{i}=+1 \mid k\right)
\end{gathered}
$$

where $P\left(S_{i}=+1 \mid k\right)$ is the probability that $S_{i}=1$ given that $k$ of its neighbors are up, which is given by ${ }^{4}$

$$
P\left(S_{i}=+1 \mid k\right)=\int_{-J(2 k / z-1)-H / \sigma}^{\infty} \phi\left(h_{i}\right) d h_{i}
$$

and $P^{*}$ is the solution when $n \rightarrow \infty$ (fixed point) of the recurrence relation:

$$
P^{(n)}=\sum_{k=0}^{z-1}\left(\begin{array}{c}
z-1 \\
k
\end{array}\right)\left[P^{(n-1)}\right]^{k}\left[1-P^{(n-1)}\right]^{z-1-k} P\left(S_{i}=+1 \mid k\right)
$$

This means that $P^{*}$ satisfies:

$$
P^{*}=\sum_{k=0}^{z-1}\left(\begin{array}{c}
z-1 \\
k
\end{array}\right)\left[P^{*}\right]^{k}\left[1-P^{*}\right]^{z-1-k} P\left(S_{i}=+1 \mid k\right)
$$

The physical meaning of $P^{*}$ is the probability that, along the up-field branch of the hysteresis loop, a spin is +1 given that a neighbor is forced to be down. For the values of $\sigma$ and $H$ for which it displays multiple fixed points only the stable fixed point obtained starting from $P^{0}=0$ will have physical meaning. For small values of $z$ the results of the Bethe lattice are in striking contrast with those of the MF case due to the neglect of environment fluctuations in this last case. The critical point $\left(H_{c}, \sigma_{c}\right)$ is absent on lattices with $z=2$ (1D model), as well as $z=3$. For $z \geqslant 4$, there is a value of the applied field $H \leqslant J / z$ where the magnetization jumps discontinuously if $\sigma<\sigma_{c}$. The size of the jump reduces with increasing $\sigma$ and vanishes as $\sigma$ approaches $\sigma_{c}$, and $H$ approaches $H_{c}=J / z$. For $\sigma>\sigma_{c}$, there is hysteresis but no discontinuity in the magnetization. The critical point for $z$ $=4$ is located at $H_{c}=J / z$ and $\sigma_{c} \approx 0.445315 \mathrm{~J}$. Figure 1 shows the phase diagram for different values of $z$. The critical points for $z=4,5, \ldots 35$ are indicated by filled circles. The discontinuity in the magnetization occurs on a field $H_{d i s}$ (indicated by the thick lines) and coercivity $(m=0)$ occurs on

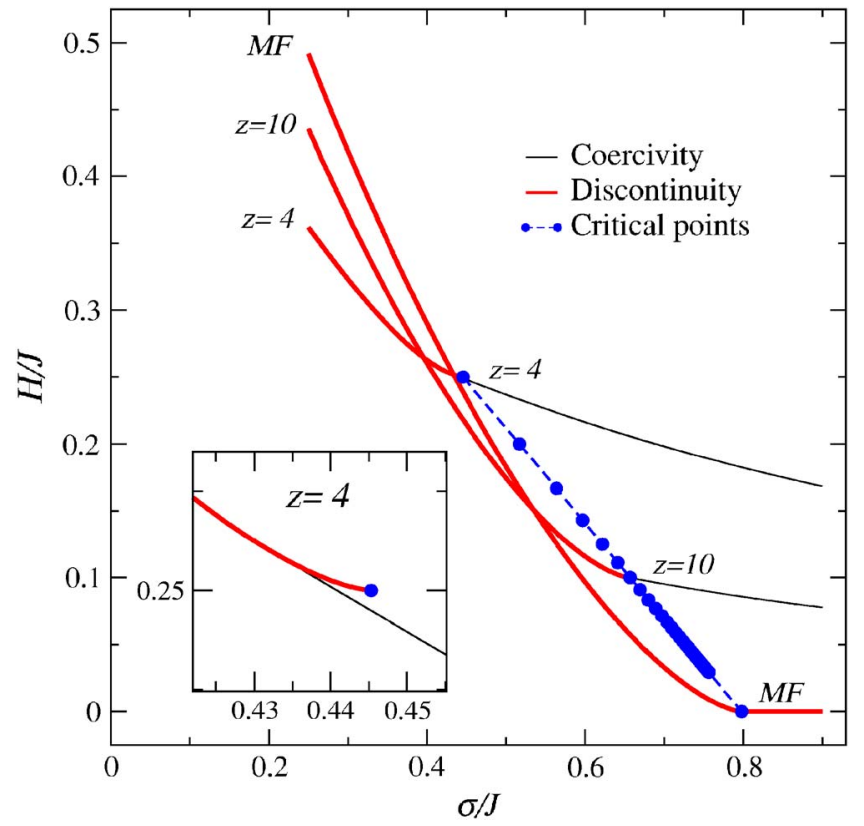

FIG. 1. (Color online) Phase diagram of the RFIM on Bethe lattices as a function of increasing $z$, compared with the MF behavior. The inset shows the details around the $z=4$ critical point.

$H_{\text {coe }}$ (thin continuous lines). The inset shows a detail of the $z=4$ case, revealing that below but close $\sigma_{c}, H_{c o e} \neq H_{d i s}$.

Although, the most important differences between the MF and B approaches are seen for small values of $z$, it is of interest to study the Bethe lattice hysteresis loops for increasing $z$. In the limit $z \rightarrow \infty$, we may expect the Bethe lattice results to approach those of the MF case. The reason is that in this limit the fluctuations in the exchange field at a site approach zero and, therefore, the model on a Bethe lattice approaches the mean-field model. This argument is not entirely transparent because unlike the mean-field model, the nearest neighbors of a site on a Bethe lattice are not nearest neighbors of each other for any $z$. However, as we see from Fig. 1, the critical point in the limit $z \rightarrow \infty$ tends to the critical point of the MF theory $H_{c}=0, \sigma_{c}=\sqrt{2 / \pi}$. Indeed, Fig. 2 shows that the entire magnetization curve tends to the MF result in the limit $z \rightarrow \infty$. Thus the expectation that the $\mathrm{B}$ results fall over the MF results in this limit is indeed born out by comparing the two numerically. The equivalence can also be shown analytically from Eq. (7) as follows: In the limit $z \rightarrow \infty$

$$
\begin{aligned}
p^{B}(h)= & \sum_{k=0}^{z}\left(\begin{array}{l}
z \\
k
\end{array}\right)\left[P^{*}\right]^{k}\left[1-P^{*}\right]^{z-k} \int_{-J(2 k / z-1)-H / \sigma}^{\infty} \phi\left(h_{i}\right) d h_{i} \\
& \Rightarrow p^{B}(h)=\int d k \delta\left(P^{*}-\frac{k}{z}\right) \int_{-J(2 k / z-1)-H / \sigma}^{\infty} \phi\left(h_{i}\right) d h_{i} \\
& \Rightarrow p^{B}(h)=\int_{-\left[J\left(2 P^{*}-1\right)+h / \sigma\right]}^{\infty} \phi\left(h_{i}\right) d h_{i}
\end{aligned}
$$

But $\left(2 P^{*}-1\right) \Rightarrow\left(2 p^{B}-1\right)=m^{B}$ in the limit $z \rightarrow \infty$. Thus the equation determining the magnetization in an applied field $H$ becomes the same in the two strategies. 


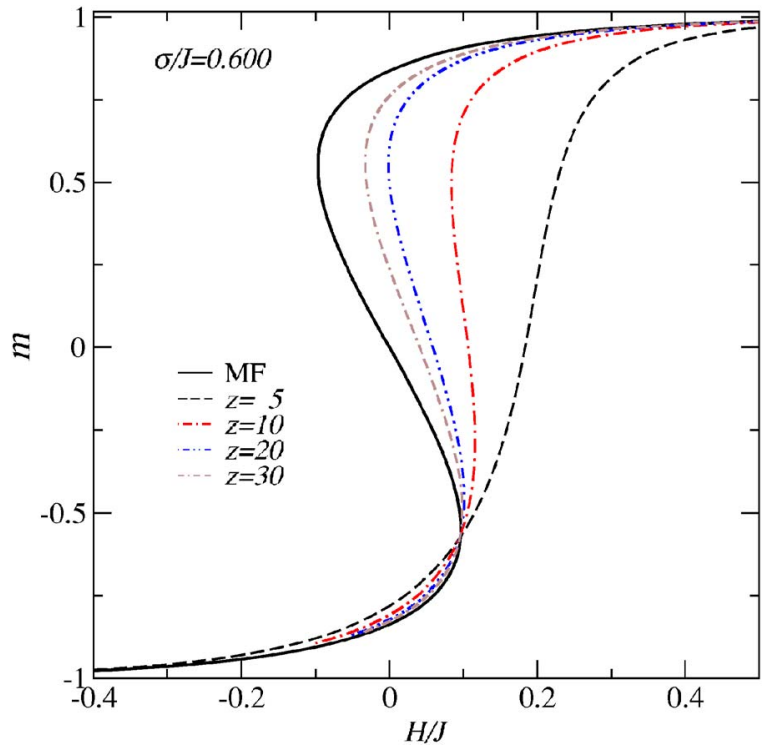

FIG. 2. (Color online) Magnetization vs field behavior for $\sigma / J$ $=0.6$ and increasing values of $z$ as indicated. The lines are compared with the MF behavior.

Let us now focus our attention on the exchange interaction along the hysteresis loop on the Bethe lattice. In a previous calculation ${ }^{4}$ the exchange interaction was computed from the two site probability $p\left(S_{i}, S_{j}\right)$. Here we will show that it can be written as a single site equation. Let us start from Eq. (11) in Ref. 4 that gives the correlation of two neighboring sites: $\left\langle S_{i} S_{j}\right\rangle$. By introducing the results of Eqs. (16), (21), (22), and (23) of the same reference one can write:

$$
\left\langle S_{i} S_{j}\right\rangle=1-4 P^{*}+4 P^{*} Q^{*}
$$

where $Q^{*}$ is given by:

$$
Q^{*}=\sum_{k=0}^{z-1}\left(\begin{array}{c}
z-1 \\
k
\end{array}\right)\left[P^{*}\right]^{k}\left[1-P^{*}\right]^{z-1-k} P\left(S_{i}=+1 \mid k+1\right)
$$

Similarly to $P^{*}, Q^{*}$ can be read as the probability that (along the increasing field branch) a spin is +1 given that a neighbor is forced to be up. Equation (12) is still a two site equation since its last term contains the double sum with two indexes reflecting the state of the $z-1$ spins (different from $S_{i}$ and $S_{j}$ ) in the neighborhoods of $S_{i}$ and $S_{j}$. Now we make use of the identity:

$$
1=\sum_{k=0}^{z}\left(\begin{array}{l}
z \\
k
\end{array}\right)\left[P^{*}\right]^{k}\left[1-P^{*}\right]^{z-k}
$$

Taking the derivative with respect to $P^{*}$ one gets

$$
P^{*}=\sum_{k=0}^{z}\left(\begin{array}{l}
z \\
k
\end{array}\right)\left[P^{* k}\right]\left[1-P^{*}\right]^{z-k} \frac{k}{z}
$$

From Eq. (13), rearranging the indices $(j=k+1)$ it is easy to obtain:

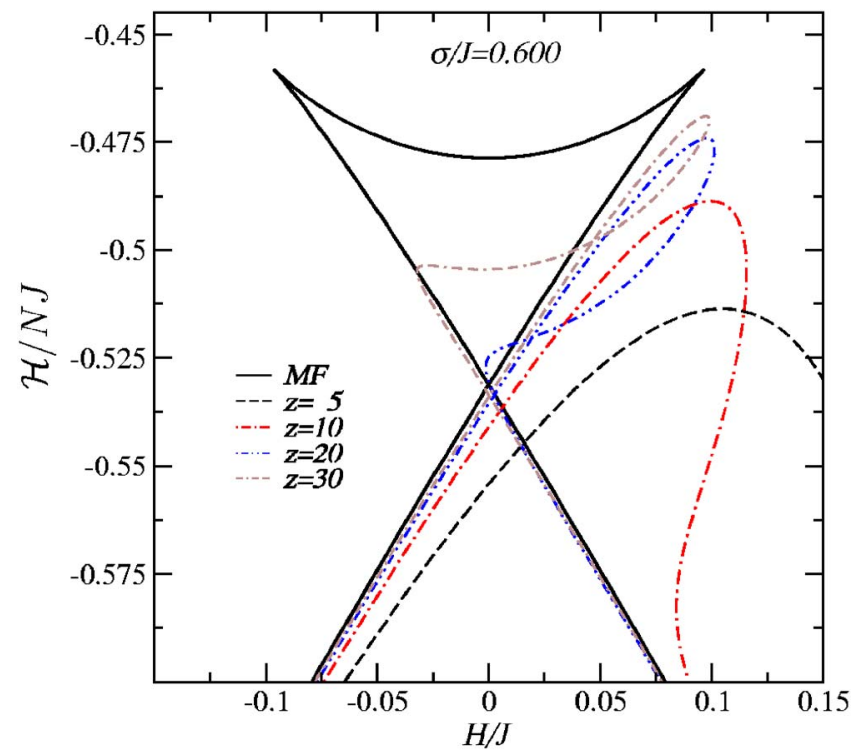

FIG. 3. (Color online) Behavior of the reduced Hamiltonian $\mathcal{H}^{B} / J N$ as a function of $H / J$ for $\sigma / J=0.6$ and increasing values of $z$, compared with the MF behavior.

$$
P^{*} Q^{*}=\sum_{k=0}^{z}\left(\begin{array}{l}
z \\
k
\end{array}\right)\left[P^{*}\right]^{k}\left[1-P^{*}\right]^{z-k} \frac{k}{z} P\left(S_{i}=+1 \mid k\right)
$$

Introducing (15) and (16) into (12), and taking into account that $\left\langle U_{e}\right\rangle / N=-\frac{J}{2}\left\langle S_{i} S_{j}\right\rangle$ one gets: ${ }^{7}$

$$
\begin{aligned}
\left\langle U_{e}\right\rangle / N= & -\frac{1}{2} J+\sum_{k=0}^{z}\left(\begin{array}{l}
z \\
k
\end{array}\right)\left[P^{*}\right]^{k}\left[1-P^{*}\right]^{z-k} \frac{2 J k}{z} \\
& \times\left[1-P\left(S_{i}=+1 \mid k\right)\right]
\end{aligned}
$$

This equation shows that the exchange energy can be computed as the energy corresponding to the saturated state plus an excess energy $(2 J / z)$ associated to each broken bond. This computation also allows to write the full average Hamiltonian $\mathcal{H}$ as a constant term plus a sum over the state of a single site environment. Figure 3 shows the behavior of $\mathcal{H} / J N$ as a function of the external field $H / J$ for $\sigma / J=0.6$ and increasing values of $z$ as indicated. As can be seen the behavior also tends to the MF behavior shown by a continuous line. It is interesting to note that even the unphysical states in the MF curve are recovered from the limit of the unphysical states of the Bethe lattice with finite $z$.

The third analysis that we want to present is that of the FORC-diagrams. Such diagrams were introduced ${ }^{6}$ in order to simplify the description of the collection of first-order reversal curves which describe the magnetization $m\left(H_{2}, H_{1}\right)$ obtained starting from saturation, adiabatically decreasing the field until $H_{1}$ and subsequently increasing the field up to $H_{2}$. The FORC-diagrams are computed by evaluating $\rho$ $=\partial^{2} m / \partial H_{1} \partial H_{2}$. This second derivative is represented as a function of $H_{u}=\left(H_{2}+H_{1}\right) / 2$ and $H_{v}=\left(H_{2}-H_{1}\right) / 2$. The fact that FORC can be computed exactly on Bethe lattices, ${ }^{3}$ which for $z \geqslant 4$ exhibit a disorder induced phase transition, 

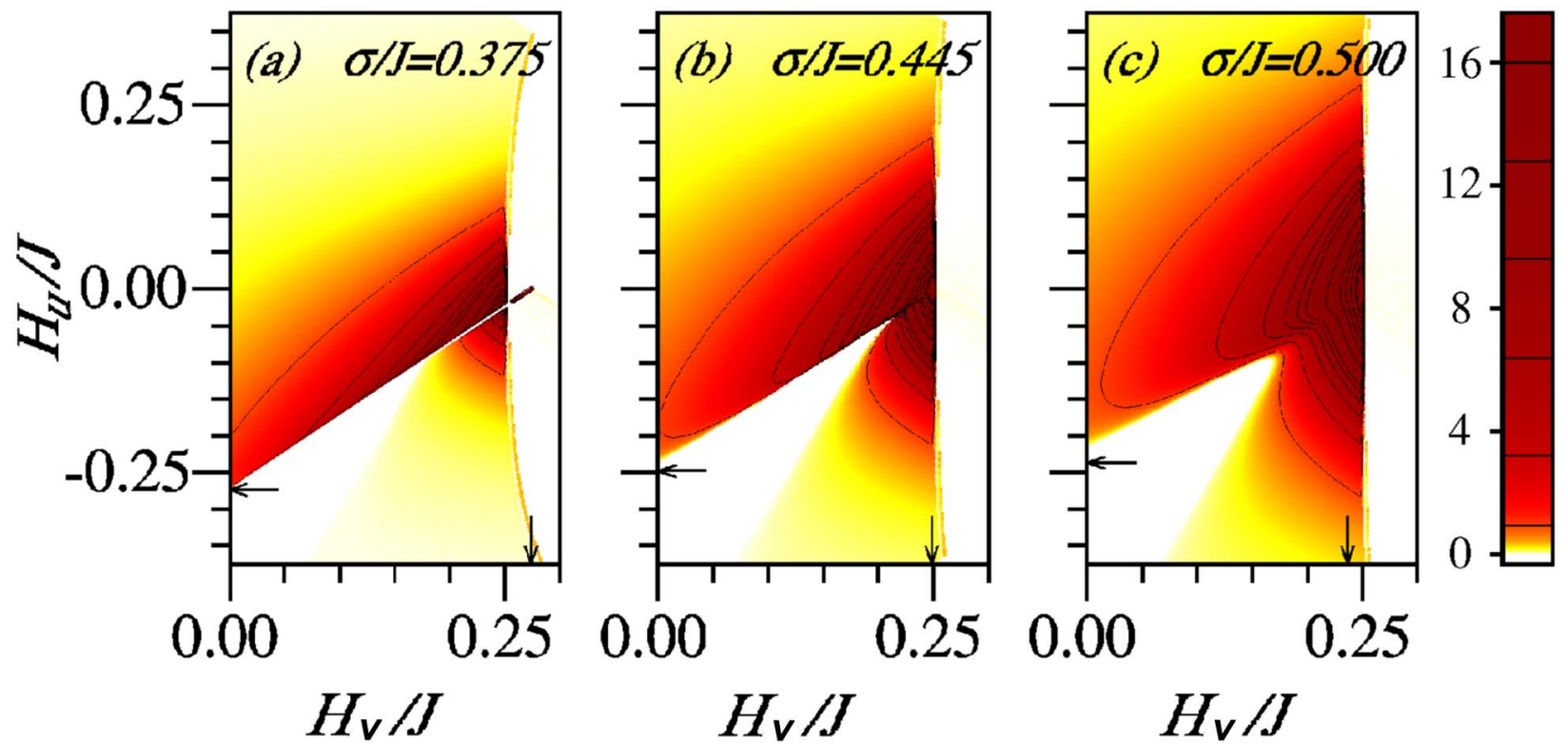

FIG. 4. (Color online) First-order reversal curves and FORC diagram for $z=4$ and three different values of $\sigma / J$, as indicated. Arrows indicate the value of the coercive field $H_{c o e} / J$ on the two axes.

allows a better understanding of some interesting features of the FORC-diagrams.

Figure 4 exhibits the FORC-diagrams corresponding to $z=4$. They have been numerically computed by evaluating the function $\rho$ in steps of $\Delta H_{1}=\Delta H_{2}=0.01 \mathrm{~J} / \mathrm{z}$ for three values of $\sigma$ as indicated. The first interesting feature, which is clearly seen in Fig. 4(c) is the existence of a well defined peak for $H_{u} \simeq 0$ and $H_{v} \simeq H_{c o e} \simeq J / z$. This indicates that the maximum variation in the slope $\partial \mathrm{m} / \partial H_{2}$ occurs around $H_{1}$ $=-H_{c o e}$ and $H_{2}=H_{c o e}$. The second remarkable property is the fact that $\rho=0$ almost everywhere for $H_{v}>J / z$. (The only exception is the infinitessimally thin "ridge" entering such a region for $\sigma<\sigma_{c}$ ). The reason is that on the Bethe lattice, FORC become independent of $H_{2}$ when $H_{2}-H_{1}>2 \mathrm{~J} / z$ since, as pointed in Ref. 3, the solutions join (and merge) the main hysteresis loop. The third feature to notice in Fig. 4 is the "valley" along an approximate line $H_{v}-H_{u} \sim J / z$. The reason for this valley is that the slope of the FORC $\partial m / \partial \mathrm{H}_{2}$ increases with decreasing $H_{1}$, is maximum when the reversing field $H_{1}$ is close to $-H_{c o e}$, and decreases again for more negative reversing fields $\left(H_{1}<-H_{c o e}\right)$. This valley is smooth for large $\sigma$ but becomes sharper when $\sigma \rightarrow \sigma_{c}$. Below $\sigma_{c}$ it transforms into a discontinuity "cliff," due to the occurence of the discontinuity in the hysteresis loop. The discontinuity in the loop also explains the existence of the infinitessimally thin ridge, when $\sigma<\sigma_{c}$ and $J / z<H_{v}<H_{d i s}$.

We acknowledge fruitful discussions with Martin-Luc Rosinberg. P.S. acknowledges the hospitality of the Universitat de Barcelona and support from Generalitat de Catalunya (Project 2004PIV2-0002). X.I. acknowledges a grant from DGI-MEC (Spain). We also acknowledge finantial support from projects MAT2004-01291 (CICyT, Spain) and SGR2001-00066 (Generalitat de Catalunya).

\footnotetext{
*Electronic address: xit@ecm.ub.es

${ }^{1}$ J. P. Sethna, K. Dahmen, S. Kartha, J. A. Krumhansl, B. W.

Roberts, and J. D. Shore, Phys. Rev. Lett. 70, 3347 (1993).

${ }^{2}$ D. Dhar, P. Shukla, and J. Sethna, J. Phys. A 30, 5259 (1997).

${ }^{3}$ P. Shukla, Phys. Rev. E 63, 027102 (2001).

${ }^{4}$ X. Illa, J. Ortín, and E. Vives, Phys. Rev. B 71, 184435 (2005).

${ }^{5}$ M. J. Alava, V. Basso, F. Colaiori, L. Dante, G. Durin, A. Magni,
}

and S. Zapperi, Phys. Rev. B 71, 064423 (2005).

${ }^{6}$ C. R. Pike, A. P. Roberts, and K. L. Verosub, J. Appl. Phys. 85, 6660 (1999).

${ }^{7}$ Note that, given the different definition of the Hamiltonian $H^{B}$ in the present paper, there is a missing $z$ factor in $U_{e}$ when comparing with Ref. 4. 